\title{
FUNCTIONS WHOSE DERIVATIVES AT ONE POINT FORM A FINITE SET $\left.{ }^{1}\right)$
}

\author{
BY
}

\section{D. NEIDLEMAN AND E. G. STRAUS}

1. Introduction. In this paper we consider families $F_{S}$ of analytic functions $f(z)$ such that the derivatives $f^{(n)}\left(z_{0}\right) ; n=0,1,2, \ldots$ all lie in a given finite set $S$ of complex numbers. Since the value of $z_{0}$ is irrelevant we shall assume $z_{0}=0$. If $S$ consists of a single element $s$ then $F_{S}=\left\{s e^{z}\right.$. We shall therefore assume $|S|>1$. The family $F_{S}$ clearly consists of entire functions of bounded exponential type. It is closed under differentiation and compact under the usual topology of uniform convergence on bounded sets. This topology is that of the space $S^{\omega}$ of sequences of elements of $S$; in other words, the topology for which the elements of a convergent family of sequences are ultimately constant. One could instead consider the Laplace transforms, thereby obtaining the family $\hat{F}_{S}$ of functions whose Taylor series expansion at 0 has only a finite number of different coefficients.

In an earlier paper [3] Sato and Straus proved that any function $f \in F_{S}$ whose derivatives at any point $z_{0} \neq 0$ form a finite set satisfies a differential equation $f^{(n)}(z)=f^{(m)}(z), n \neq m$ so that the set of derivatives at every point is finite. In $\S 2$ we investigate the value distributions of the functions in $F_{S}$ under more general conditions. In particular we show that the closure $\langle f\rangle\left(z_{0}\right)$ of the set $\left\{f^{(n)}\left(z_{0}\right) \mid n\right.$ $=0,1,2, \ldots\}$, if infinite, has the same cardinality (which is either $\boldsymbol{N}_{0}$ or $2 \boldsymbol{k}_{0}$ ) at every point $z_{0} \neq 0$ and that for all $z_{0}$, except for a totally disconnected perfect set, the continuous mapping from $F_{S}$ to the set of values $F_{S}\left(z_{0}\right)$ is one-to-one.

In $\S 3$ we examine the structure of the sets $\langle f\rangle$ in greater detail, by looking at the successive derived sets and the order type beyond which the derived sets are the same. This investigation is essentially independent of function theory and could equally concern the behavior of the fractional parts of numbers $b^{n} x$ where $b>1$ is an integer and $x$ a real number.

A rather striking result in Theorem 3.13 shows that denumerably infinite closed subsets of the unit interval which are closed under multiplication $(\bmod 1)$ by an integer $b>1$ must contain an infinite closed set of rational numbers.

Throughout the paper we shall use the following notation:

$$
\begin{aligned}
m & =\min _{s \in S \backslash\{0\}}|s|, & M & =\max _{s \in S}|s|, \\
\delta & =\min _{s_{1}, s_{2} \in S, s_{1} \neq s_{2}}\left|s_{1}-s_{2}\right|, & \Delta & =\max _{s_{1}, s_{2} \in S}\left|s_{1}-s_{2}\right| .
\end{aligned}
$$

Received by the editors July 25,1968 .

( $\left.{ }^{1}\right)$ The preparation of this paper was supported in part by Grant GP-8622. 
1.3. Definition. Let $G$ be a (closed) set, then for every ordinal $\alpha$ we define $G_{\alpha}$ inductively by $G_{0}=G, G_{1}=G^{\prime}, G_{\alpha+1}=G_{\alpha}^{\prime}$, the derived set of $G_{\alpha}$ and if $\lambda$ is a limit ordinal then

$$
G_{\lambda}=\bigcap_{\alpha<\lambda} G_{\alpha}
$$

1.4. Definition. The topological order type of a (closed) set $G$ is the least ordinal $\alpha=\alpha(G)$ such that $G_{\alpha+1}=G_{\alpha}$.

1.5. Definition. If $G$ is a set of functions then $G\left(z_{0}\right)=\left\{g\left(z_{0}\right) \mid g \in G\right\}$.

1.6. Definition. $N_{S}=\left\{z \mid f(z)=0\right.$ for some $\left.f \in F_{S}, f \neq 0\right\}$.

1.7. Definition. A point $z_{0}$ is a representation point of $F_{S}$ if the mapping $F_{\mathrm{S}} \rightarrow F_{\mathrm{S}}\left(z_{0}\right)$ is one-to-one.

1.8. Definition. If $A, B$ are sets of numbers then $A-B=\{a-b \mid a \in A, b \in B\}$.

1.9. Definition. For every $f \in F_{S}$ the set $\langle f\rangle$ is the closure of the set $\left\{f^{(n)} \mid n=0\right.$, $1,2, \ldots\}$.

\section{The value distributions of functions in $F_{S}$.}

2.1. TheOrem. Let $f \in F_{S}, f$ not identically 0 , and let $n_{0}$ denote the number of zeroes of $f$ at 0 while $n(r)$ denotes the number of zeros, $z$, of $f$ which satisfy $0<|z|<r$. Then

$$
n(r) \leqq \log _{2}\left(1+\frac{M}{m}\left(e^{2 r}-1\right)\right)
$$

and

$$
\begin{aligned}
n^{*}(r) & =n_{0}+n(r) \\
& \leqq n_{0}+\log _{2}\left(1+\frac{M}{m} \frac{2 r}{n_{0}+1}\left(1+\frac{2 r}{n_{0}+2}+\frac{4 r^{2}}{\left(n_{0}+2\right)\left(n_{0}+3\right)}+\cdots\right)\right)
\end{aligned}
$$

Proof. Inequality (2.2) is a consequence of (2.3) and the fact that

$$
\frac{2 r}{n_{0}+1}\left(1+\frac{2 r}{n_{0}+2}+\frac{4 r^{2}}{\left(n_{0}+2\right)\left(n_{0}+3\right)}+\cdots\right) \leqq 2 r\left(1+\frac{2 r}{2}+\frac{4 r^{2}}{2 \cdot 3}+\cdots\right)=e^{2 r}-1 .
$$

Now, if $f \in F_{S}$ has a zero of order $n_{0}$ at 0 then

$$
f(z)=\sum_{n=n_{0}}^{\infty} \frac{c_{n} z^{n}}{n !}=\frac{z^{n_{0}}}{n_{0} !}\left(c_{n_{0}}+\frac{c_{n_{0}+1}}{n_{0}+1} z+\cdots\right)=\frac{z^{n_{0}}}{n_{0} !} g(z)
$$

with $c_{n} \in S, c_{n_{0}} \neq 0$. Thus, if $z_{1}, \ldots, z_{n(r)}$ are the zeros of $f$-and hence of $g$-in the punctured disk $0<|z|<r$, then by Jensen's formula

$$
\frac{1}{2 \pi} \int_{0}^{2 \pi} \log \left|g\left(2 r e^{i \theta}\right)\right| d \theta=\log |g(0)|+\log \frac{(2 r)^{n(2 r)}}{\left|z_{1} \cdots z_{n(2 r)}\right|}
$$


or, as a result of $(2.4)$,

$$
\begin{aligned}
\log 2^{n(r)} & \leqq \log \frac{(2 r)^{n(r)}}{\left|z_{1} \cdots z_{n(r)}\right|} \leqq \log \frac{(2 r)^{n(2 r)}}{\left|z_{1} \cdots z_{n(2 r)}\right|} \\
& =\frac{1}{2 \pi} \int_{0}^{2 \pi} \log \left|\frac{g\left(2 r e^{i \theta}\right)}{g(0)}\right| d \theta \\
& \leqq \log \left(1+\frac{M}{m}\left(\frac{2 r}{n_{0}+1}+\frac{4 r^{2}}{\left(n_{0}+1\right)\left(n_{0}+2\right)}+\cdots\right)\right),
\end{aligned}
$$

which yields (2.3).

Of course if $0 \notin S$ then $n_{0}=0$.

As a result of Theorem 2.1 we see that there exists a neighborhood, $U$, of 0 such that no nonzero function of $F_{S}$ has a zero different from 0 in $U$. We can find the best possible bounds for the radius of $U$ that can be expressed in terms of $m$ and $M$.

2.7. THEOREM. If $f \in F_{S}, f \not \equiv 0$ then $f(z) \neq 0$ for

$$
0<|z|<\log (1+m / M) .
$$

If $f$ has a zero of order $n_{0}$ at 0 then $f(z) \neq 0$ for $0<|z|<r_{S}\left(n_{0}\right)$ where $r=r_{S}\left(n_{0}\right)$ is the positive solution of

$$
m\left(r^{n_{0}} / n_{0} !\right)=M\left(e^{r}-1-r / 1 !-\cdots-r^{n_{0}} / n_{0} !\right) .
$$

In particular,

$$
r_{\mathrm{S}}\left(n_{0}\right)>\frac{m\left(n_{0}+1\right)\left(n_{0}+2\right)}{m\left(n_{0}+1\right)+M\left(n_{0}+2\right)}
$$

Proof. The equations $z \neq 0$ and

lead to

$$
f(z)=\sum_{n=n_{0}}^{\infty} \frac{c_{n} z^{n}}{n !}=\frac{z^{n_{0}}}{n_{0} !}\left(c_{n_{0}}+\frac{c_{n_{0}+1}}{n_{0}+1} z+\cdots\right)=0
$$

$$
\begin{aligned}
m & \leqq\left|c_{n_{0}}\right|=\left|\frac{c_{n_{0}+1}}{n_{0}+1} z+\frac{c_{n_{0}+2}}{\left(n_{0}+1\right)\left(n_{0}+2\right)} \dot{z}^{2}+\cdots\right| \\
& \leqq M\left(e^{|z|}-1-\frac{|z|}{1 !}-\cdots-\frac{|z|^{n_{0}}}{n_{0} !}\right) \cdot \frac{n_{0} !}{|z|^{n_{0}}} .
\end{aligned}
$$

Since the right side of (2.11) is an increasing function of $|z|$, it follows that $|z|$ $\geqq r_{S}\left(n_{0}\right)$ where $r_{S}\left(n_{0}\right)$ is the solution of (2.9). To verify (2.10) it is clear that it holds when $r \geqq n_{0}+2$. If $r<n_{0}+2$ then

$$
\begin{aligned}
m & =M\left(e^{r}-1-\cdots-\frac{r^{n_{0}}}{n_{0} !}\right) \frac{n_{0} !}{r^{n_{0}}}=M \frac{r}{n_{0}+1}\left(1+\frac{r}{n_{0}+2}+\frac{r^{2}}{\left(n_{0}+2\right)\left(n_{0}+3\right)}+\cdots\right) \\
& \leqq M \frac{r}{n_{0}+1} \sum_{k=0}^{\infty}\left(\frac{r}{n_{0}+2}\right)^{k}=M \frac{r}{n_{0}+1} /\left(1-\frac{r}{n_{0}+2}\right)
\end{aligned}
$$

leads to (2.10). 
For $n_{0}=0$ equation (2.9) becomes $m=M\left(e^{r}-1\right)$ leading to

$$
r_{S}(0)=\log \left(1+\frac{m}{M}\right)<\frac{m}{M}
$$

and for each $n_{0}>0$ we have

$$
\frac{m}{M}<\frac{m\left(n_{0}+1\right)\left(n_{0}+2\right)}{m\left(n_{0}+1\right)+M\left(n_{0}+2\right)}<r_{S}\left(n_{0}\right) .
$$

Thus $|z| \geqq r_{S}\left(n_{0}\right)$ for any $n_{0}$ implies $|z| \geqq r_{S}(0)$ which verifies (2.8).

2.12. Corollary. If $f_{n} \in F_{S}, f_{n} \not \equiv 0, f_{n} \rightarrow 0$ and $f_{n}\left(z_{n}\right)=0, z_{n} \neq 0$, then $z_{n} \rightarrow \infty$.

Proof. This is an immediate consequence of (2.10) since $r_{S}\left(n_{0}\right) \rightarrow \infty$ as $n_{0} \rightarrow \infty$ and, as $f_{n} \rightarrow 0$, the multiplicity, $n_{0}$, of the zero of $f_{n}$ at 0 tends to infinity.

2.13. CoRollary. For every value, a, there is a neighborhood $U$ of 0 so that no $f \in F_{S}$ which is not identically equal to a attains the value $a$ in $U \backslash\{0\}$. The radius of $U$ can be chosen as

$$
\log (1+m(a) / M) \text { where } m(a)=\min \{|s-a| \mid s \in S, s \neq a\},
$$

2.14. Corollary. Every point $z$ satisfying

$$
0<|z|<\log (1+\delta / \Delta)
$$

is a representation point of $F_{S}$.

Proof. A point $z_{0}$ fails to be a representation point of $F_{S}$ only if $f\left(z_{0}\right)=g\left(z_{0}\right)$ for two different functions $f, g \in F_{S}$, in other words, if the nonzero function $f-g \in F_{S-S}$ has a zero at $z_{0}$. The corollary now follows from the application of Theorem 2.7 to $F_{S-s}$.

2.16. THEOREM. The set $N_{S}$ of zeros of the nonzero elements of $F_{S}$ is a totally disconnected and nowhere dense closed set. If $0 \in S$ then 0 is an isolated point of $N_{S}$. In any case $N_{S} \mid\{0\}$ is perfect.

Proof. The fact that 0 is isolated follows from Theorem 2.7. If $z_{n} \in N_{S}$ and $z_{n} \rightarrow z_{0} \neq 0$ then there exist nonzero functions $f_{n} \in F_{S}$ so that $f_{n}\left(z_{n}\right)=0$ and since $F_{S}$ is compact there is a convergent subsequence $f_{n_{i}} \rightarrow f_{0} \in F_{S}$. Since $\left\{z_{n}\right\}$ is bounded, it follows from Corollary 2.12 that $f_{0} \neq 0$. On the other hand

$$
f_{0}\left(z_{0}\right)=\lim f_{n_{i}}\left(z_{0}\right)=\lim \left(f_{n_{i}}\left(z_{0}\right)-f_{n_{i}}\left(z_{n_{i}}\right)\right)=0 .
$$

Thus $z_{0} \in N_{S}$ and $N_{S}$ is closed.

The set $F_{S}$ is perfect so that for every $f_{0} \in F_{S} \mid\{0\}$ with $f_{0}\left(z_{0}\right)=0, z_{0} \in N_{S} \mid\{0\}$ there exists a sequence $f_{n} \in F_{S}, f_{n} \neq f_{0}, f_{n} \rightarrow f_{0}$. By Rouche's theorem all $f_{n}$ sufficiently near to $f_{0}$ have a zero, $z_{n}$, arbitrarily close to $z_{0}$. On the other hand, $z_{n} \neq z_{0}$ for large $n$, since otherwise $f_{n}-f_{0}$ would have a zero at $z_{0}$ in contradiction to Corollary 2.12. Thus $z_{0}$ is a limit point of $N_{S}$ and $N_{S} \mid\{0\}$ is perfect. 
Now assume that $N_{S}$ contains a continuum $C$ (compact connected set consisting of more than one point). If we divide $C$ into $|S|^{n}$ (not necessarily disjoint) closed sets of zeros of functions $f \in F_{S}$ with fixed values of $f^{(v)}(0) ; \nu=0,1, \ldots, n-1$ then at least one of these sets must contain a subcontinuum $C_{1}$. Let the corresponding subset of $F_{S}$ be $F_{1}$.

Now according to Corollary 2.12 we can choose $n$ so large that no two distinct functions which have the same first $n$ derivatives can have the same value at any point of $C_{1}$, since otherwise their difference would approximate 0 and at the same time have a zero in the bounded set $C_{1}$. Thus for sufficiently large $n$ the set $F_{1}$ must consist of a single function $f_{1}$ which vanishes on $C_{1}$ and therefore vanishes identically-a contradiction.

2.17. Corollary. The correspondence $f \leftrightarrow f(z)$ is a homeomorphism between $F_{S}$ and $F_{S}(z)$ whenever $z \notin N_{S-s}$. That is, $F_{S}$ and $F_{S}(z)$ are homeomorphic for all $z$ except for a nowhere dense totally disconnected perfect set of nonrepresentation points and the origin.

Proof. The continuity of the mapping $F_{S} \rightarrow F_{S}(z)$ is obvious. Now assume $f_{n}(z) \rightarrow w_{0}$, then, by the compactness of $F_{S}$, there exists a subsequence $f_{n_{1}} \rightarrow f_{0}$ with $f_{0}(z)=w_{0}$. Now, unless $f_{n} \rightarrow f_{0}$, there would exist another subsequence $f_{n_{i}} \rightarrow g_{0} \neq f_{0}$. Then $g_{0}(z)=w_{0}$ and $\left(f_{0}-g_{0}\right)(z)=0$ so that $z \in N_{S-S}$ contrary to hypothesis.

2.18. THEOREM. The number of different functions of $F_{S}$ which attain the same value at a point $z_{0} \neq 0$ is less than

$$
\mathscr{N}\left(z_{0}, S\right)=|S|^{\left|z_{0}\right|(1+\Delta / \delta)-1} .
$$

Proof. If the number of functions is at least $\mathscr{N}\left(z_{0}, S\right)$ then there must exist two functions $f, g \in F_{S}$ with $f\left(z_{0}\right)=g\left(z_{0}\right)$ and $f^{(v)}(0)=g^{(v)}(0)$ for $\nu=0,1, \ldots, n_{0}-1$ where $n_{0} \geqq\left|z_{0}\right|(1+\Delta / \delta)-1$ so that $(f-g)\left(z_{0}\right)=0$ and

$$
\left|z_{0}\right| \leqq \frac{\delta\left(n_{0}+1\right)}{\delta+\Delta}<\frac{\delta\left(n_{0}+1\right)\left(n_{0}+2\right)}{\delta\left(n_{0}+1\right)+\Delta\left(n_{0}+2\right)},
$$

which contradicts (2.10) as applied to the set $S-S$, that is with $m$ replaced by $\delta$ and $M$ replaced by $\Delta$.

2.19. Corollary. If $G$ is an infinite subset of $F_{S}$ then $\left|G\left(z_{0}\right)\right|=|G|$ for every $z_{0} \neq 0$.

The continuous finite-to-one map $G \rightarrow G\left(z_{0}\right)$ of any $G \subset F_{S}$ preserves more than the cardinality of infinite $G$, it also preserves the topological order types of denumerable closed sets.

2.20. Lemma. For any $G \subset F_{S}$ the derived sets satisfy $G_{1}\left(z_{0}\right)=\left(G\left(z_{0}\right)\right)_{1}$ for any $z_{0} \neq 0$. 
Proof. Assume $g_{n}\left(z_{0}\right) \rightarrow w \in\left(G\left(z_{0}\right)\right)_{1}$ where $\left(g_{n}\left(z_{0}\right)\right)$ is an infinite sequence with $g_{n} \in G$. Then there exists a convergent subsequence $g_{n_{i}} \rightarrow g_{0} \in G_{1}$ so that $w=g_{0}\left(z_{0}\right)$ $\in G_{1}\left(z_{0}\right)$. Thus $\left(G\left(z_{0}\right)\right)_{1} \subset G_{1}\left(z_{0}\right)$. Now assume $g_{n} \rightarrow g_{0} \in G_{1}$ where $\left(g_{n}\right)$ is an infinite sequence. Then, by Theorem 2.13 , the sequence $\left(g_{n}\left(z_{0}\right)\right)$ contains infinitely many distinct elements so that $\lim g_{n}\left(z_{0}\right)=g_{0}\left(z_{0}\right) \in\left(G\left(z_{0}\right)\right)_{1}$. Thus $\left(G\left(z_{0}\right)\right)_{1} \supset G_{1}\left(z_{0}\right)$.

2.21. Corollary. For each ordinal $\alpha$ and any $G \subset F_{S}$ we have $G_{\alpha}\left(z_{0}\right)=\left(G\left(z_{\mathrm{c}}\right)\right)_{\alpha}$ for any $z_{0} \neq 0$.

Proof. By Lemma 2.20 the corollary holds for $\alpha=1$. If the corollary holds for $\alpha$ then

$$
\left(G\left(z_{0}\right)\right)_{\alpha+1}=\left(\left(G\left(z_{0}\right)\right)_{\alpha}\right)_{1}=\left(G_{\alpha}\left(z_{0}\right)\right)_{1}=\left(G_{\alpha}\right)_{1}\left(z_{0}\right)=G_{\alpha+1}\left(z_{0}\right) .
$$

If $\lambda$ is a limit ordinal and the corollary holds for all $\alpha<\lambda$ then

$$
\left(G\left(z_{0}\right)\right)_{\lambda}=\bigcap_{\alpha<\lambda}\left(G\left(z_{0}\right)\right)_{\alpha}=\bigcap_{\alpha<\lambda} G_{\alpha}\left(z_{0}\right)=\left(\bigcap_{\alpha<\lambda} G_{\alpha}\right)\left(z_{0}\right)=G_{\lambda}\left(z_{0}\right) .
$$

Thus the corollary holds by transfinite induction.

2.22. Corollary. For every $G \subset F_{S}$ and every $z_{0} \neq 0$ we have $\alpha\left(G\left(z_{0}\right)\right) \leqq \alpha(G)$.

Proof. If $G_{\alpha+1}=G_{\alpha}$ then

$$
\left(G\left(z_{0}\right)\right)_{\alpha+1}=G_{\alpha+1}\left(z_{0}\right)=G_{\alpha}\left(z_{0}\right)=\left(G\left(z_{0}\right)\right)_{\alpha} .
$$

2.23. TheOREM. For every $G \subset F_{S}$ the topological order type $\alpha(G)$ is denumerable and $G_{\alpha(G)}$ is the set of all condensation points of $G$, which is the maximal perfect set in the closure $\bar{G}$ of $G$. In particular, if the closure of $G$ is denumerable, then $G_{\alpha(G)}=\varnothing$.

Proof. Since $\left(G_{\alpha(G)}\right)^{\prime}=G_{\alpha(G)}$ it follows that the set is perfect. Since every perfect subset of $\bar{G}$ belongs to all derived sets of $G$ it follows that it is contained in $G_{\alpha(G)}$. Thus $G_{\alpha(G)}$ is the maximal perfect subset of $\bar{G}$. The set of all condensation points of $\bar{G}$ is clearly perfect and every point of a nonempty perfect set is a condensation point. Now if $\alpha(G)$ were nondenumerable then the set $\bar{G} \mid G_{\alpha(G)}$ would be nondenumerable. By the separability of $F_{S}$ there would be a neighborhood $U$ of $G_{\alpha(G)}$ so that $|\bar{G}| U \mid>\mathcal{\aleph}_{0}$. Hence $\bar{G} \mid U$ would have condensation points not in $G_{\alpha(G)}$, a contradiction.

2.24. THEOREM. If $G \subset F_{S}$ is closed and denumerable then $\alpha(G)=\alpha\left(G\left(z_{0}\right)\right)$ for every $z_{0} \neq 0$.

Proof. If $G$ is finite then trivially $\alpha(G)=\alpha\left(G\left(z_{0}\right)\right)=1$. If $\beta+1<\alpha(G)$ then $G_{\beta}$ is infinite and hence, according to Corollary $2.19, G_{\beta}\left(z_{0}\right)$ is infinite. Since $G_{\beta}\left(z_{0}\right)$ is compact it follows that $\left(G\left(z_{0}\right)\right)_{\beta+1} \neq \varnothing$ so that $\alpha\left(G\left(z_{0}\right)\right)>\beta+1$. Now the topological order type of a denumerable compact set cannot be a limit ordinal since the intersection of a nested family of nonempty compact sets is nonempty. Hence we 
can write $\alpha\left(G\left(z_{0}\right)\right)=\beta+1$, and $\beta+1<\alpha(G)$ would lead to the contradiction $\beta+1<\alpha\left(G\left(z_{0}\right)\right)$.

3. On denumerable sets $\langle f\rangle$. In this section we wish to consider the closure of the set of all derivatives of a function $f$ in $F_{S}$, in particular in the exceptional cases in which $\langle f\rangle$ is denumerable. It will be convenient to identify the function $f$ with the sequence $\left(f^{(n)}(0)\right)$ whose elements are in $S$. Such sequences in turn can be identified with numbers in the interval $[0,1]$ by introducing a basis $b=|S|$ and a mapping $\beta\left(s_{v}\right)=\nu-1 ; \nu=1,2, \ldots, b$ of the elements of $S$ onto the integers $0,1, \ldots$, $b-1$. We then let

$$
f \leftrightarrow\left(f^{(n)}(0)\right) \rightarrow x(f)=\sum_{n=0}^{\infty} \beta\left(f^{(n)}(0)\right) b^{-n-1} .
$$

The last mapping is not quite one-to-one because of the ambiguity in the expansion of $b$-adic fractions. The differentiation operation for $f$ corresponds to the shift operation for the sequence and to multiplication by $b(\bmod 1)$ for the real number. Thus $\langle f\rangle$ corresponds to the closed set invariant under multiplication by $b(\bmod 1)$ which is generated by a single point $x(f)$ of $[0,1]$.

3.2. Definition. To a set $G \subset F_{S}$ we assign the measure $\mu(G)=\mu(x(G))$, where $x(G)=\{x(g) \mid g \in G\}$, provided the latter measure exists.

3.3. THEOREM. For almost all (in the sense of $\mu$ ) functions of $F_{S}$ we have $\langle f\rangle=F_{S}$.

Proof. If $x(f)$ is normal to the base $b$ then $x(\langle f\rangle)=[0,1]=x\left(F_{S}\right)$ and $\langle f\rangle=F_{S}$. Of course $\langle f\rangle$ may equal $F_{S}$ even when $x(f)$ is not normal.

It will be convenient to see that the structure of the sets of $F_{S}$ which we wish to examine is the same as the structure of the sets we get for $S$ with 2 elements.

3.4. Lemma. Given $G \subset F_{S}$ then there exists $a G^{*} \subset F_{\{0,1\}}$ which is homeomorphic to $G$.

Proof. To each $s \in S$ we associate the block $B(s)$ of length $\beta(s)+2$ consisting of $\beta(s)$ digits 0 and digits 1 at the ends. To each sequence $g \in G$ we now associate the sequence $g^{*} \in F_{\{0,1\}}$ obtained by replacing each element $s$ of $g$ by the block $B(s)$. This one-to-one association between $F_{S}$ and $F_{S}^{*} \subset F_{\{0,1\}}$ is clearly a homeomorphism.

The correspondence established in Lemma 3.4 will, in general, associate a set $G^{*}$ which is not closed under differentiation to a given $G \subset F_{S}$ which is closed under differentiation. However the behavior of $G^{*}$ under differentiation can be determined.

3.5. Lemma. If $G \subset F_{S}$ is closed under differentiation, then the closure under differentiation $G^{* *}$ of $G^{*} \subset F_{\{0,1\}}$ is

$$
G^{* *} \subseteq G_{(0)}^{*} \cup G_{(1)}^{*} \cup \cdots \cup G_{(b+1)}^{*}
$$

where $G_{(0)}^{*}=G^{*}$, each $G_{(i)}^{*}$ is the union of a finite number of sets each homeomorphic 
to a subset of $G$ where the closures of these subsets are disjoint, and the closures of $G_{(i)}^{*}$ and $G_{(j)}^{*}$ are disjoint for $i \neq j$.

Proof. Let $G_{(i)}^{*}$ for $i>0$ consist of the sequences in $G^{*}$ preceded by a digit 1 which in turn is preceded by $i-1$ digits 0 . These are clearly all the sequences of $F_{\{0,1\}}$ obtained by successive differentiation of the elements of $G^{*}$. The closures of $G_{(i)}^{*}$ and $G_{(j)}^{*}$ are disjoint for $i \neq j$ since the initial blocks of length $b$ are distinct. Finally each $G_{(i)}^{*}$ corresponds to those elements in $G$ whose initial digit is $s_{\beta}$ with $\beta \geqq i$. In other words there are $b-i+1$ subsets of $G$ with distinct initial digits, the union of whose homeomorphic images in $F_{\{0,1\}}$ is $G_{(i)}^{*}$.

3.7. TheOREM. For each $G \subset F_{S}$ there is a $G^{*} \subset F_{\{0,1\}}$ so that $\alpha(G)=\alpha\left(G^{*}\right)$. If $G$ is closed under differentiation then there is $a G^{* *} \subset F_{(0,1)}$ so that $G^{* *}$ is closed under differentiation and $\alpha(G)=\alpha\left(G^{* *}\right)$. If $G$ is also closed under integration (that is, if every element of $G$ is the derivative of an element of $G)$ then $G^{* *}$ is closed under integration. For every $f \in F_{S}$ there is an $f^{*} \in F_{\{0,1\}}$ so that $\alpha(\langle f\rangle)=\alpha\left(\left\langle f^{*}\right\rangle\right)$.

Proof. The first statement is an immediate consequence of Lemma 3.4 since $\alpha(G)$ is a topological invariant. The second statement follows from Lemma 3.5 and

$$
\begin{aligned}
\alpha(G) & =\alpha\left(G^{*}\right) \leqq \alpha\left(G^{* *}\right)=\alpha\left(G^{*} \cup G_{(1)}^{*} \cup \cdots \cup G_{(b+1)}^{*}\right) \\
& =\max \left\{\alpha\left(G^{*}\right), \alpha\left(G_{(1)}^{*}\right), \ldots, \alpha\left(G_{(b+1)}^{*}\right)\right\}=\alpha\left(G^{*}\right) .
\end{aligned}
$$

It is clear that $G^{* *}$ is closed under integration whenever $G$ is.

Finally, if we define $f^{*}$ by $\{f\}^{*}=\left\{f^{*}\right\}$ then $\langle f\rangle^{* *}=\left\langle f^{*}\right\rangle$ so that the last statement of the theorem is a special case of $\alpha\left(G^{* *}\right)=\alpha(G)$.

3.8. Lemma. For every denumerable closed set $G \subset F_{\{0,1\}}$ there exists a closed $G^{\sim} \subset F_{\{0,1,2\}}$, which is closed under integration, so that

$$
\alpha\left(G^{\sim}\right)=\alpha(G)+1, \quad G_{\alpha(G)}^{\tilde{a}}=\left\{2 e^{z}\right\} .
$$

If $G$ is closed under differentiation then so is $G^{\sim}$.

Proof. Let $G^{\sim}$ consist of the sequences in $G$ preceded by an arbitrary number of 2 's and the element $2 e^{z}$. Then for every $\beta \leqq \alpha(G)$ the set $G_{\tilde{\beta}}$ consists of the sequences in $G_{\beta}$ preceded by an arbitrary number of 2's and the element $2 e^{z}$. In particular $G_{\alpha(G)}^{\tilde{\alpha}}=\left\{2 e^{z}\right\}$ and $\alpha\left(G^{\sim}\right)=\alpha(G)+1$.

3.9. Lemma. For each sequence of denumerable closed sets $G_{n} \subset F_{\{0,1\}}$ there exists a closed. $G^{\sim} \subset F_{\{0,1,2\}}$ such that

$$
\alpha\left(G^{\sim}\right)=\sigma+1 \text { where } \sigma=\sup _{n} \alpha\left(G_{n}\right) .
$$

If the $G_{n}$ are closed under differentiation then there exists a closed $G^{\approx} \subset F_{\{0,1,2\}}$ so that

$$
\alpha\left(G^{\approx}\right)=\sigma+2, \quad G_{\sigma+1}^{\approx}=\left\{2 e^{z}\right\}
$$

and $G^{\approx}$ is closed under differentiation and integration. 
Proof. If $\sigma=\alpha\left(G_{m}\right)$ for some $m$ then the lemma results from Lemma 3.8. If not, let $G_{n}^{\tilde{n}}$ consist of the elements of $G_{n}$ preceded by $n$ digits 2 . Then $G_{\tilde{n}}^{\tilde{n}}$ is obviously homeomorphic to $G_{n}$ and $G_{n}^{\tilde{n}} \cap G_{m}^{\tilde{m}}=\varnothing$ for $n \neq m$. Let $G^{\sim}=\bigcup_{n} G_{n}^{\tilde{n}}$ $\cup\left\{2 e^{z}\right\}$. Then $G^{\sim}$ is closed, since $\lim g_{n_{i}}=2 e^{z}$ if $g_{n_{i}} \in G_{n_{i}}$ and $\lim n_{i}=\infty$. Now for every $\beta<\sigma$ we have $G_{\beta} \tilde{\beta}=\bigcup_{n}\left(G_{n}^{\tilde{n}}\right)_{\beta} \cup\left\{2 e^{z}\right\}$ so that $G_{\sigma}^{\tilde{\sigma}}=\left\{2 e^{z}\right\}$ and $G_{\tilde{\sigma}+1}^{\tilde{1}}=\varnothing$.

To prove the second part of the lemma associate to each $G_{n}$ the set $G^{\approx} \subset F_{\{0,1,2\}}$ consisting of $2 e^{z}$ and the elements obtained from the sequences $g_{n} \in G_{n}$ by inserting $n$ terms 2 between any two consecutive terms of $g_{n}$ and an arbitrary number of terms 2 before the first term of $g_{n}$. Then $G_{n}^{\approx}$ is closed and closed under integration. Since $G_{n}$ is closed under differentiation then so is $G_{n}^{z}$. Now

$$
G_{n}^{\approx}=\left\{2 e^{z}\right\} \cup G_{n 0}^{\approx} \cup G_{n 1}^{\approx} \cup \cdots
$$

where the second index indicates the number of terms 2 at the beginning of the sequences. The $G_{n i}^{\approx}$ are disjoint and homeomorphic to $G_{n}$. Let $\alpha_{n}=\alpha\left(G_{n}\right)$. Then $\alpha_{n}$ is not a limit ordinal and $\left(G_{n}\right)_{\alpha_{n}-1}$ consists of a finite number of (eventually periodic) sequences. Thus $\left(G_{n}^{z}\right)_{\alpha_{n}-1}$ consists of $2 e^{z}$ and a finite number of (eventually periodic) sequences preceded by blocks of 2's of arbitrary length. Hence $\left(G_{n}^{*}\right)_{\alpha_{n}}$ $=\left\{2 e^{z}\right\}$ and $\alpha\left(G_{n}^{\approx}\right)=\alpha\left(G_{n}\right)+1$.

Now let $G^{\approx}=\bigcup_{n} G_{n}^{\approx} \cup H$ where $H$ consists of all sequences of 2's with at most one digit 0 or 1 in an arbitrary place. It is clear that any convergent sequence $\left(g_{n_{1}}\right)$ with $n_{1}<n_{2}<\cdots, g_{n_{1}} \in G_{n_{i}}^{\approx}$ must converge to an element of $H$ and that every element of $H$ is such a limit.

Now for each $\beta<\sup _{n} \alpha\left(G_{n}\right)=\sup _{n} \alpha\left(G_{n}^{\approx}\right)$ we have

$$
G^{\approx}=\bigcup_{n}\left(G_{n}^{\approx}\right)_{\beta} \cup H
$$

since for all sufficiently large $n$ the set $\left(G_{n}\right)_{\beta}$ is infinite and contains elements whose initial block consists of an arbitrary number of 2's followed by 0 or 1 followed by $n$ terms 2. If $\sigma=\sup _{n} \alpha\left(G_{n}\right)$ we get $\left(G_{n}^{\approx}\right)_{\sigma}=\varnothing$ and $G_{\sigma}^{\approx}=H$. Now $G_{\sigma+1}^{\approx}=H_{1}=\left\{2 e^{z}\right\}$ so that $G_{\sigma+2}^{\approx}=\varnothing$ and $\alpha\left(G^{z}\right)=\sigma+2$.

3.10. Lemma. If $G$ is a denumerable compact set then $\alpha(G)$ is not a limit ordinal. If, in addition, $G \subset F_{S}$ and $G$ is closed under differentiation then $\alpha(G)$ is not the successor of a limit ordinal.

Proof. If $\lambda$ is a limit ordinal and $G_{\beta} \neq \varnothing$ for $\beta<\lambda$ then $G_{\lambda}=\bigcap_{\beta<\lambda} G_{\beta} \neq \varnothing$ since it is the intersection of a nested family of nonempty compact sets.

If $G \subset F_{S}$ and $\alpha(G)=\lambda+1$, where $\lambda$ is a limit ordinal, then $G_{\lambda}$ is finite, consisting of a finite number of eventually periodic sequences. Let $f \in G_{\lambda}$ be purely periodic of period $p$. For each $\beta<\lambda$ the set $G_{\beta}$ contains a sequence $f_{\beta n} \rightarrow f$ and $f_{\beta n} \notin G_{\lambda}$. By the periodicity of $f$ we have $f_{\beta n}^{(p k+l)} \rightarrow f^{(l)}$ for each $l=0,1, \ldots, p-1$ and $k=0,1$, $2, \ldots$ Pick $p N$ so large that no two elements of $G_{\lambda}$ have the same initial segment of length $p N$ and choose $f_{\beta n}$ so that it has the same initial segment of length $p N$ as $f$. Let the first term in $f_{\beta n}$ which differs from the corresponding term of $f$ be the 
$(p N+p k+l)$ th one. Then $f_{\beta n}^{(p k+l)}$ agrees with $f^{(l)}$ precisely in the first $p N$ terms and disagrees in $(p N+1)$ st term. In other words, each $G_{\beta}, \beta<\lambda$ contains an $f_{\beta}$ which agrees with some $f^{(l)}$ in the first $p N$ terms but disagrees in the $(p N+1)$ st term. Since the $f^{(l)}$ form a finite set there is one $l$ which corresponds to a cofinal sequence of $\beta$ 's and hence to all $\beta<\lambda$. Similarly since there are only $|S|-1$ choices for the $(p N+1)$ st term there is a cofinal sequence of $\beta$ 's with the same $(p N+1)$ st digit. Since $F_{S}$ is compact, these $f_{\beta}$ have a convergent subsequence converging to $f^{*} \in G_{\lambda}$ whose initial block of $p N+1$ digits disagrees with the initial blocks of the elements of $G_{\lambda}$, a contradiction.

3.11. THEOREM. For each denumerable ordinal $\alpha$ which is not a limit ordinal there exists a closed denumerable $G \subset F_{\{0,1\}}$ which is closed under integration such that $\alpha(G)=\alpha$. If $\alpha$ is not the successor of a limit ordinal then the set $G$ can be chosen to be closed under differentiation. The restrictions on $\alpha$ are necessary. For each of these $\alpha$ we can pick $G \subset F_{\{0,1,2\}}$ so that $G_{\alpha-1}=\left\{2 e^{z}\right\}$.

Proof. We first construct $G \subset F_{\{0,1,2\}}$. For $\alpha=1$ we can pick $G=\left\{2 e^{z}\right\}$. If the theorem holds for $\alpha$ then it holds for $\alpha+1$ by Lemma 3.8. If the theorem holds for all $\alpha<\lambda$, where $\lambda$ is a limit ordinal, then it holds for $\lambda+1$ and $\lambda+2$ respectively by Lemma 3.9. Thus the theorem holds for all denumerable ordinals. The necessity of the restrictions on $\alpha$ is the result of Lemma 3.10. The fact that all constructions can be made in $F_{\{0,1\}}$ was proved in Theorem 3.7.

3.12. Lemma. For every $f \in F_{S}$ the set $\langle f\rangle_{1}$ is closed under differentiation and integration. If $\langle f\rangle$ is infinite then so is $\langle f\rangle_{1}$.

Proof. Let $g \in\langle f\rangle_{1}$, then $g=\lim f^{\left(n_{i}\right)}$, where $n_{1}<n_{2}<\cdots$. Thus $g^{\prime}=\lim f^{\left(n_{i}+1\right)}$ and the sequence $\left(f^{\left(n_{i}-1\right)}\right)$ has a convergent subsequence which converges to an integral of $g$.

If $\langle f\rangle_{1}$ is finite it can be closed under integration only if all its elements are purely periodic. If $\langle f\rangle$ is infinite then $f$ is not eventually periodic. Therefore $f$ must contain infinitely many blocks of consecutive digits of some length, $l$, which are not initial blocks of any element of $\langle f\rangle_{1}$. Since the number of different blocks is finite, there must be some block of length $l$ which occurs infinitely often in $f$ but does not occur in any element of $\langle f\rangle_{1}$. This contradicts the compactness of $\langle f\rangle$.

3.13. TheOREM. A denumerably infinite closed subset of $F_{S}$ which is closed under differentiation contains an infinite closed subset of solutions of differential equations of the form $f^{(n+p)}=f^{(n)}$ with fixed $p$.

Equivalently, a denumerably infinite closed set of sequences with elements in a finite set which is closed under the shift operation contains an infinite closed subset of eventually periodic sequences with fixed period $p$.

Equivalently, a denumerably infinite closed subset of the interval $[0,1]$, which is closed under multiplication by an integer $b>1$ modulo 1 , contains an infinite closed subset of rational points whose denominators are bounded multiples of powers of $b$. 
Proof. If we call the set in question $G$, then according to Theorem 3.10 we have $\alpha(G)=\beta+2$ where $G_{\beta+1}$ consists of a finite number of eventually periodic sequences. Let $p$ be the common period of the elements of $G_{\beta+1}$ and let all elements of $G_{\beta+1}$ be periodic from the $N$ th term on.

Now $G_{\beta+1}$ contains $\langle g\rangle_{1}$ for every $g \in G_{\beta}$ and thus, by Lemma 3.12, the set $\langle g\rangle$ is finite for every $g \in G_{\beta}$. In other words all elements of $G_{\beta}$ are eventually periodic. If $g \in G_{\beta}$ does not have period $p$ then there exists an $M \geqq N$ so that $g^{(M+p)}(0) \neq g^{(M)}(0)$. Thus the initial block of length $N+p$ of $g^{(M-N)}$ does not agree with the initial block of any element of $G_{\beta+1}$. The number of elements of $G_{\beta}$ whose initial block of a fixed length disagrees with the initial blocks of all elements of $G_{\beta+1}$ must be finite due to the compactness of $G_{\beta}$. Hence there exists a finite number $g_{1}, g_{2}, \ldots, g_{n}$ of elements of $G_{\beta}$ such that every $g \in G_{\beta}$ of period different from $p$ has derivative of some order equal to some $g_{i}$. Thus, if we let $P$ be the least common multiple of $p$ and the periods of $g_{1}, \ldots, g_{n}$, then every element of $G_{\beta}$ has eventual period $P$. The set $G_{\beta}$ satisfies the conditions of the theorem.

It is worth noting that closure and closure under differentiation without the hypothesis of denumerability do not imply the existence of any periodic elements. For example, there exist sequences $f \in F_{\{0,1,2\}}$ so that no two adjacent blocks of digits are equal [1]. Thus $\langle f\rangle$ can contain no periodic elements. According to Theorem 3.13 we can infer that $|\langle f\rangle|=2^{x_{0}}$.

3.14. TheOREM. For every denumerable ordinal $\alpha$ which is different from 2, a limit ordinal, or the successor of a limit ordinal, there exists an $f \in F_{S}$ so that $\langle f\rangle$ is denumerable and $\alpha(\langle f\rangle)=\alpha$. The restrictions on $\alpha$ are necessary.

Proof. If $\alpha=1$ we can choose $f$ periodic so that $\langle f\rangle$ is finite and $\langle f\rangle_{1}=\varnothing$. If $\alpha>1$ write $\alpha=\beta+2$. If $\beta$ is finite we can pick, by Theorem 3.11, a denumerable closed $G \subset F_{\{0,1\}}$ which is closed under differentiation and integration and has $\alpha(G)=\beta$. If $\beta$ is infinite but not a limit ordinal we can pick by Theorem 3.4 a denumerable closed $G \subset F_{\{0,1\}}$ which is closed under differentiation and integration and has $\alpha(G)=\beta+1$. If $\beta$ is a limit ordinal we pick a sequence of denumerable closed $G_{n} \subset F_{\{0,1\}}$ which are closed under differentiation and satisfy $\sup \alpha\left(G_{n}\right)=\beta$. We then let $G \subset F_{\{0,1,2\}}$ be the set $G^{\approx}$ constructed in Lemma 3.9.

Now let $G=\left\{g_{n} \mid n=1,2, \ldots\right\}$ and let $g_{n m}$ be the initial block of length $m$ of $g_{n}$. We define the sequence of blocks $B_{n}$ by $B_{1}=g_{11}$ and if $B_{n}=g_{i j}$ then

$$
\begin{array}{ll}
B_{n+1}=g_{i+1, j} & \text { if } i<j, \\
B_{n+1}=g_{1, j+1} & \text { if } i=j .
\end{array}
$$

Thus $\left(B_{1}, B_{2}, B_{3}, \ldots\right)=\left(g_{11}, g_{12}, g_{22}, g_{13}, g_{23}, g_{33}, \ldots\right)$.

Now define $f$ as the sequence consisting of the blocks $B_{n}$ in succession with $n$ digits 2 between $B_{n}$ and $B_{n+1}$. Then $\langle f\rangle_{1}$ consists of the sequences of $G$ preceded by an arbitrary number of 2's and infinite sequences of 2's preceded by an arbitrary block $g_{i j}$. The latter sequences occur in $\langle f\rangle_{1}$ since $G$ is closed under integration 
and thus every $g_{i j}$ occurs infinitely often as the terminal block of $B_{n}$. Thus $\langle f\rangle_{2}$ consists of the sequences of $G_{1}$ preceded by an arbitrary number of 2's, of $G$ and of $2 e^{z}$. Hence for each $1+\gamma\left\langle\alpha(G)\right.$ the set $\langle f\rangle_{2+\gamma}$ consists of the sequences of $G_{1+\gamma}$ preceded by an arbitrary number of 2's, of $G_{y}$ and of $2 e^{z}$.

In case $\beta$ is finite we therefore have

$$
\langle f\rangle_{\beta+1}=\langle f\rangle_{1+\beta}=\langle f\rangle_{2+(\beta-1)}=G_{\beta-1} \cup\left\{2 e^{z}\right\}
$$

which is finite nonempty so that $\alpha(\langle f\rangle)=\beta+2$.

In case $\beta$ is infinite but not a limit ordinal we have

$$
\langle f\rangle_{\beta+1}=\langle f\rangle_{2+(\beta+1)}=\left\{2 e^{z}\right\}
$$

so that again $\alpha(\langle f\rangle)=\beta+2$.

If $\beta$ is a limit ordinal then

$$
\langle f\rangle_{\beta+1}=G_{\beta+1}=\left\{2 e^{z}\right\}
$$

so that $\alpha(\langle f\rangle)=\beta+2$.

4. Open questions and concluding remarks. We have seen that there are functions in $F_{S}$ which satisfy particularly simple differential equations of the form $f^{(m)}=f^{(n)}$, $m \neq n$. It is easy to see that every solution in $F_{S}$ of a linear differential equation with constant coefficients has eventually periodic derivatives, since the solutions of a linear recurrence equation with elements in the finite set $S$ form a periodic sequence [2]. It is easy to show that the result holds for functions of $F_{S}$ which satisfy linear differential equations with polynomial coefficients. Probably more is true.

4.1. ConjeCture. A solution in $F_{S}$ of an algebraic differential equation with constant coefficients satisfies $f^{(m)}=f^{(n)}$ for some $m \neq n$.

It is possible to generalize these investigations to functions of several variables. The conditions for the theorems in $\$ 2$ become more complicated.

\section{REFERENCES}

1. G. A. Hedlund and Marston Morse, Unending chess, symbolic dynamics and a problem in semigroups, Duke Math. J. 11 (1944), 1-7.

2. Christer Lech, A note on recurring series, Ark. Mat. 2 (1953), 417-421.

3. D. Sato and E. G. Straus, Generalized interpolation by analytic functions, J. Math. Sci. 1 (1966), 53-76.

UNiversity OF CALIFornia, Los Angeles, California 